\title{
RATIONAL MODELING OF MULTIVARIATE MULTI-FIDELITY DATA
}

\author{
Elizabeth Rita Samuel ${ }^{1}$,Dirk Deschrijver ${ }^{1}$, Luc Knockaert $^{1}$, Tom Dhaene $^{1}$,Annie Cuyt ${ }^{2}$ \\ ${ }^{1}$ Ghent University - iMinds \\ Gaston Crommenlaan 8, 9050 Ghent, Belgium \\ e-mail: \{dirk.deschrijver,luc.knockaert,tom.dhaene\} @intec.ugent.be \\ ${ }^{2}$ University of Antwerp \\ Middelheimlaan 1, 2020 Antwerp, Belgium \\ e-mail: $\{$ annie.cuyt $\}$ ua.ac.be
}

Keywords: Rational functions, multi-fidelity data, modeling, barycentric interpolation.

\begin{abstract}
Accurate multi-fidelity modeling is of high importance in the present day engineering design process. It allows to model computationally expensive simulations at a reduced cost by combining simulations with variable fidelity levels. In this paper, a novel algorithm is proposed to build multivariate models from variable fidelity simulations using rational functions. The modeling is based on high-fidelity data and low-fidelity data that is sampled over a parameter space of interest. The former is assumed to be computationally expensive and sparse, whereas the latter is cheaper to obtain but comes at a lower accuracy. It is shown that accurate rational models can be built at a reduced cost by combining these types of data. The effectiveness of the algorithm is applied to several examples and confirmed by numerical results.
\end{abstract}




\section{INTRODUCTION}

Nowadays, there is a growing need for accurate behavioral models in many engineering applications and research disciplines. These models mimic the input-output behavior of a system, which is usually characterized by multiple parameters (also called variables) that describe the physical properties of the system over a certain parameter range of interest. The availability of such models has proven very useful for tasks like optimization, design space exploration, virtual prototyping and sensitivity analysis. In order to build models, a representative set of data samples must be collected by performing simulations. Unfortunately, as systems are becoming increasingly complex, the computational cost associated with simulations gets prohibitively high and limits the complexity of systems that can be handled.

The modeling of data with different levels of fidelity can be very useful in areas where the computational effort of an analysis is substantial, such as in computational fluid dynamics (CFD) and finite element (FE) analysis. The term "fidelity" refers to the rigorousness of the simulations and the level of detail by which an underlying system is represented. For example, depending on the mesh refinement of a system, physics-based simulations can be performed to collect data having various accuracy levels. On one hand, a dense mesh discretization leads to time-consuming simulations used to generate very accurate data, henceforth called highfidelity (HF) data. On the other hand, a coarse mesh discretization significantly speeds up the simulations and generates data that captures the overall trend, although it is often less precise. This type of data is called low-fidelity (LF) data. It was shown in $[1,2,3]$ that accurate models can be built at a reduced cost by combining data with different fidelity levels, hereby combining the best of both worlds.

A well known algorithm for multi-fidelity modeling is the auto-regressive co-kriging algorithm, which is a geostatistical technique that originates from mining and geology $[4,5]$. It models the HF data exactly and accounts for spatial correlation in the LF data. In the case of linear time-invariant systems that are described by a set of differential equations, however, it is preferable to model the data with rational functions because this model type corresponds better to the underlying nature of the system [6]. The modeling of data using rational functions has been studied intensively in literature, and several algorithms are available. The reader is referred to $[7,8,9,10]$ for a survey and analysis of univariate methods. More details on algorithms for multivariate rational modeling are provided in $[11,12,13,14]$. In these works, it is assumed that the data originates from a single source and that all available data samples have a comparable level of fidelity. This is one of the key aspects that will be addressed in this paper.

This paper proposes a novel algorithm that builds multivariate models from multi-fidelity data using rational functions. To this end, it makes use of the barycentric interpolation algorithm which has excellent numerical properties $[15,16]$. First, a multivariate rational model that exactly interpolates the HF data is built using barycentric interpolation. Second, the remaining model coefficients are calculated such that the (calibrated) LF data is approximated in a leastsquares (LS) sense. The algorithm is applied to model the quality factor and inductance of a spiral inductor. Numerical results confirms the effectiveness of the approach.

\section{ALGORITHM OUTLINE}

The goal of the algorithm is to identify a multivariate rational function $\mathcal{R}$ that approximates the behavioral response of an underlying system $f$. For notational convenience, it will be assumed that this system depends on 2 parameters ( $\alpha$ and $\beta$ ), although the extension to multivari- 
ate responses is analogous and conceptually straightforward.

$$
\mathcal{R}(\alpha, \beta)=\frac{N(\alpha, \beta)}{D(\alpha, \beta)}=\frac{\sum_{i=0}^{I} \sum_{j=0}^{J} c_{i j} \alpha^{i} \beta^{j}}{\sum_{i=0}^{I} \sum_{j=0}^{J} \tilde{c}_{i j} \alpha^{i} \beta^{j}} .
$$

To ensure that $\mathcal{R}$ matches $f$ as closely as possible, some data is needed to estimate the unknown model coefficients $c$ and $\tilde{c}$. To this end, the system $f$ is simulated using different levels of fidelity, resulting in two disjoint sets of data

$$
\begin{aligned}
\mathrm{HF} & =\left\{\left(\alpha_{k}^{H}, \beta_{k}^{H}, f^{H}\left(\alpha_{k}^{H}, \beta_{k}^{H}\right)\right)\right\}_{k=1}^{K_{H}} \\
\mathrm{LF} & =\left\{\left(\alpha_{i}^{L}, \beta_{i}^{L}, f^{L}\left(\alpha_{i}^{L}, \beta_{i}^{L}\right)\right)\right\}_{i=1}^{K_{L}}
\end{aligned}
$$

The HF data is sampled on a sparse grid of $K_{H}=M \times N$ points that are equidistantly spaced in the $(\alpha, \beta)$ parameter space. The LF data comprises $K_{L}$ data samples that can be scattered throughout the parameter space. Taking into account the computational cost of simulations, it is assumed that the HF data is sparse when compared to the LF data $\left(K_{H}<<K_{L}\right)$. To ensure that both types of data are compatible, the ratio between HF and LF data is computed as a calibration factor to scale the LF data accordingly $[17,18,19]$. Then, a two-step modeling approach is proposed:

1. Compute a rational model that exactly interpolates all the HF data by using a barycentric interpolation formula.

2. Calculate the coefficients of the model in such a way that the (calibrated) LF data is approximated in a LS sense.

It is assumed that the HF data is too sparse in order to generate a global model with sufficient accuracy. However, by exploiting the information that is contained within the LF data, it is possible to control the behavior of model $\mathcal{R}$ inbetween the HF data samples. By combining both types of data, a model $\mathcal{R}$ with good overall accuracy is obtained.

\section{RATIONAL MODELING ALGORITHM}

In order to facilitate a mixture of interpolation and LS approximation, the multivariate rational function $\mathcal{R}$ in (1) is represented in a different functional form [20]. To fix the barycentric representation of the rational function $[15,16]$, the input values of the HF data are used as the interpolation points such that $\mathcal{R}\left(\alpha_{k}^{H}, \beta_{k}^{H}\right)=f^{H}\left(\alpha_{k}^{H}, \beta_{k}^{H}\right)$ for $k=1, \ldots, K_{H}$ and for any choice of non-zero barycentric weights $\left(\boldsymbol{w}=\left[w_{k}\right] \in \mathbb{R}^{K_{H} \times 1}\right.$ with $\left.w_{k} \neq 0\right)$.

$$
\mathcal{R}(\alpha, \beta, \boldsymbol{w})=\frac{N(\alpha, \beta, \boldsymbol{w})}{D(\alpha, \beta, \boldsymbol{w})}=\frac{\sum_{k=1}^{K_{H}} \frac{w_{k}}{\left(\alpha-\alpha_{k}^{H}\right)\left(\beta-\beta_{k}^{H}\right)} f^{H}\left(\alpha_{k}^{H}, \beta_{k}^{H}\right)}{w_{0}+\sum_{k=1}^{K_{H}} \frac{w_{k}}{\left(\alpha-\alpha_{k}^{H}\right)\left(\beta-\beta_{k}^{H}\right)}} .
$$

The choice of $\boldsymbol{w}$ in (4) allows one to enforce additional properties to the model. In this work, these additional degrees of freedom are exploited in such a way that the interpolation model also approximates the LF data in a LS sense. This corresponds to finding optimal value for 
the weight $\boldsymbol{w}$ such that sum of the squared residuals is minimized. Note that a residual is the difference between the actual function value $f$ and the model response.

$$
\arg \min _{\boldsymbol{w}} \sum_{i=1}^{K_{L}}\left|\frac{N\left(\alpha_{i}^{L}, \beta_{i}^{L}, \boldsymbol{w}\right)}{D\left(\alpha_{i}^{L}, \beta_{i}^{L}, \boldsymbol{w}\right)}-f^{L}\left(\alpha_{i}^{L}, \beta_{i}^{L}\right)\right|^{2}
$$

Unfortunately, problem (5) is non-linear and therefore it becomes difficult to solve in a fast and accurate way. Instead, Levi proposes to solve a linear approximation of the problem [21].

$$
\arg \min _{\boldsymbol{w}} \sum_{i=1}^{K_{L}}\left|N\left(\alpha_{i}^{L}, \beta_{i}^{L}, \boldsymbol{w}\right)-f^{L}\left(\alpha_{i}^{L}, \beta_{i}^{L}\right) D\left(\alpha_{i}^{L}, \beta_{i}^{L}, \boldsymbol{w}\right)\right|^{2}
$$

This way, an estimate for $\boldsymbol{w}$ can be found by solving a linear set of LS equations (7) of the form $\boldsymbol{A} \boldsymbol{x}=\boldsymbol{b}$ using the matlab operator ' $\backslash$ ' (mldivide). Note that this problem is overdetermined because $K_{L}>>K_{H}$. One can the weight $w_{0}=1$ in (4) without loss of generality.

$$
\begin{gathered}
\boldsymbol{A}=\left[a_{i k}\right] \in \mathbb{R}^{K_{L} \times K_{H}} \quad \text { and } \quad \boldsymbol{x}=\boldsymbol{w} \in \mathbb{R}^{K_{H} \times 1} \quad \text { and } \quad \boldsymbol{b}=\left[b_{i}\right] \in \mathbb{R}^{K_{L} \times 1} \\
\text { where } a_{i k}=\frac{f^{H}\left(\alpha_{k}^{H}, \beta_{k}^{H}\right)-f^{L}\left(\alpha_{i}^{L}, \beta_{i}^{L}\right)}{\left(\alpha_{i}^{L}-\alpha_{k}^{H}\right)\left(\beta_{i}^{L}-\beta_{k}^{H}\right)} \text { and } b_{i}=f^{L}\left(\alpha_{i}^{L}, \beta_{i}^{L}\right) .
\end{gathered}
$$

It is known that Levi's formulation is biased and not equivalent to (6), because a weighting factor $1 /\left|D\left(\alpha_{i}^{L}, \beta_{i}^{L}, \boldsymbol{w}\right)\right|^{2}$ is omitted in each term of the summation. To improve the accuracy of the model, the estimated weights $w_{k}$ are refined iteratively by applying the Sanathanan-Koerner (SK) iteration [9] with explicit weighting as illustrated in [22].

$$
\arg \min _{\boldsymbol{w}} \sum_{i=1}^{K_{L}}\left|\frac{N^{t}\left(\alpha_{i}^{L}, \beta_{i}^{L}, \boldsymbol{w}\right)-f^{L}\left(\alpha_{i}^{L}, \beta_{i}^{L}\right) D^{t}\left(\alpha_{i}^{L}, \beta_{i}^{L}, \boldsymbol{w}\right)}{D^{t-1}\left(\alpha_{i}^{L}, \beta_{i}^{L}, \boldsymbol{w}\right)}\right|^{2} .
$$

This iteration multiplies each row in the LS matrix with an explicit weighting function $1 /\left|D^{t-1}(\alpha, \beta, \boldsymbol{w})\right|^{2}$, which is based on Levi's initial (iteration step 0 ) or the previous (iteration step $t-1)$ estimate of the denominator. This way, updated values of the weights can be found in successive steps $t=0, \ldots, T$. The final model is then $\mathcal{R}^{T}(\alpha, \beta, \boldsymbol{w})=N^{T}(\alpha, \beta, \boldsymbol{w}) / D^{T}(\alpha, \beta, \boldsymbol{w})$. Although the solution of (8) is not guaranteed to converge to the solution of (5) either, the SK iteration is known to provide good results for sufficiently high signal-to-noise ratios [22].

\section{MODEL COEFFICIENT RELAXATION}

In (7) it was chosen to set $w_{0}=1$, because numerator and denominator can be divided by the same constant value without loss of generality. In some cases, this can lead to a poor convergence of the SK iteration. This problem was investigated in [23] and it was proposed to make $w_{0}$ a variable in the LS problem. This gives rise to a modified set of equations $\left[\begin{array}{ll}\boldsymbol{A} & -\boldsymbol{b}\end{array}\right] \boldsymbol{x}=0$. To avoid the trivial null solution, one additional equation is added to the LS problem.

$$
\sum_{i=1}^{K_{L}}\left(\sum_{k=1}^{K_{H}} \frac{w_{k}}{\left(\alpha_{i}^{L}-\alpha_{k}^{H}\right)\left(\beta_{i}^{L}-\beta_{k}^{H}\right)}+w_{0}\right)=K_{L}
$$


Equation (9) is scaled in relation to the size of $f^{L}\left(\alpha^{L}, \beta^{L}\right)$ in the LS problem by a scaling factor

$$
\text { scale }=\frac{\left\|\boldsymbol{f}^{L}\left(\alpha^{L}, \beta^{L}\right)\right\|_{2}}{K_{L}}
$$

Note that this additional equation does not impose any further constraints on the LS problem other than preventing the trivial null solution. The reader is referred to [23] for a more details.

\section{NUMERICAL RESULTS}

In this example, the rational modeling of a three turn spiral inductor is considered as shown in Figure 1. The dielectric has a thickness of $300 \mu \mathrm{m}$, a relative dielectric constant $\epsilon_{r}=9.6$ and a loss tangent $\tan \delta=0.0002$. The conductivity of the metallic layers is equal to $\sigma=5.8 \times 10^{7}$ $S / \mathrm{m}$. The spacing between conductors is equal to $10 \mu \mathrm{m}$.

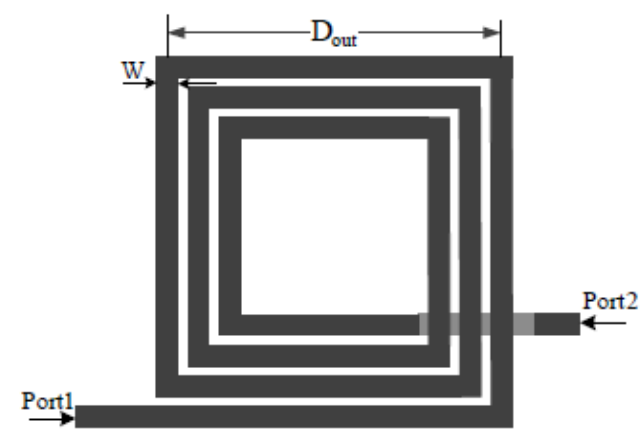

Figure 1: Layout of the spiral inductor (Top View).

The width of the conductors $W$ and the outer length $D_{\text {out }}$ are considered as the 2 input parameters $(\alpha$ and $\beta)$ and their corresponding ranges are shown in Table 1. The rational modeling technique is used to model the inductance $L$ and quality factor $Q$ of the spiral inductor at frequency $f r e q=2.4 \mathrm{GHz}$. The $L$ and $Q$ are calculated from the simulated admittance parameters (Y-parameters) as in (11). The Y-parameters describe the electrical behavior of linear electrical networks where the terminal currents can be expressed in terms of the terminal voltages [24].

Table 1: Range of the design parameters.

\begin{tabular}{lll}
\hline Parameter & Width $(W)$ & Outer length $\left(D_{\text {out }}\right)$ \\
\hline Lower bound & $4 \mu \mathrm{m}$ & $140 \mu \mathrm{m}$ \\
Upper bound & $15 \mu \mathrm{m}$ & $210 \mu \mathrm{m}$ \\
\hline
\end{tabular}

$$
\begin{aligned}
L\left(W, D_{\text {out }}\right) & =\frac{1}{2 \pi f r e q} \Im m \frac{1}{Y_{12}\left(\text { freq }, W, D_{\text {out }}\right)} \\
Q\left(W, D_{\text {out }}\right) & =\frac{\Im m\left(Y_{11}\left(\text { freq }, W, D_{\text {out }}\right)\right)}{\Re e\left(Y_{11}\left(\text { freq }, W, D_{\text {out }}\right)\right)} .
\end{aligned}
$$




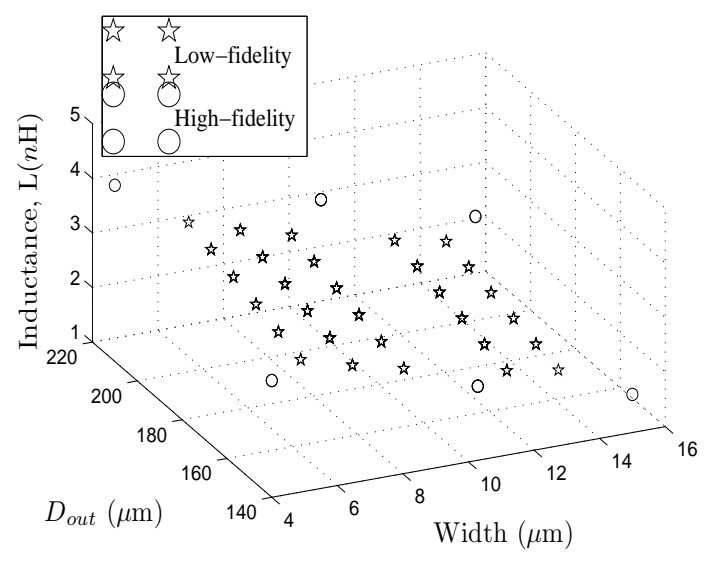

(a) High-fidelity and low-fidelity samples

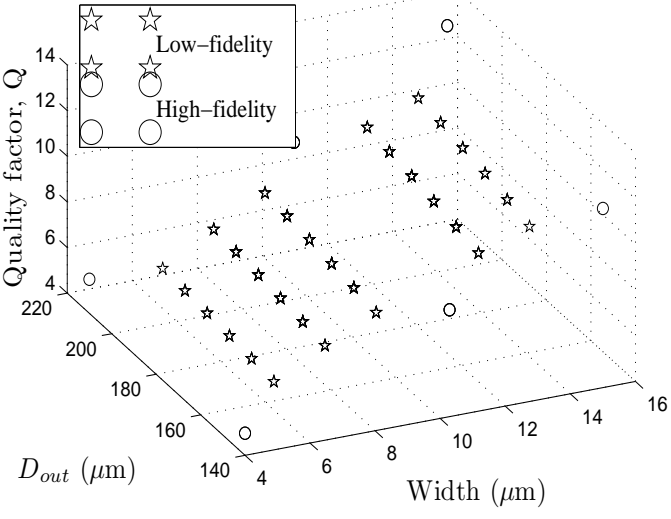

(b) High-fidelity and low-fidelity samples

Figure 2: HF and LF data samples considered for the modeling using rational function.

The Y-parameters are obtained from the full-wave electromagnetic simulator Agilent Advanced Design System1 (ADS) Momentum ${ }^{1}$, whereas the LF data are obtained using circuit schematic ADS simulations. The circuit schematic simulations are based on analytical formulas for the electrical behavior of the spiral inductor, whereas the electromagnetic simulations are based on the solution of Maxwell's equations, which is computationally more expensive. A set of LF and a set of HF data samples are collected on a grid of $5 \times 6\left(W, D_{\text {out }}\right)$ and $3 \times 2\left(W, D_{\text {out }}\right)$ respectively for the frequency $2.4 \mathrm{GHz}$ to build the rational model. The computation time to get $\mathrm{LF}$ and $\mathrm{HF}$ admittance parameters at one sample point in the $2 \mathrm{D}\left(W, D_{\text {out }}\right)$ parameter space is roughly equal to $0.0301 \mathrm{~s}$ and $0.2541 \mathrm{~s}$, respectively. Note that these timing results were performed on an Intel ${ }^{(R)}$ Core $^{(T M)} 2$ Duo P8700 $2.53 \mathrm{GHz}$ machine with 2 GB RAM and has been implemented in Matlab R2012b on the Windows 7 platform. For checking the accuracy of the model, relative error is used, which is defined as follows, where $f$ represents either $L$ or $Q$.

$$
\text { Rel.Err }=\frac{\left|f\left(W, D_{\text {out }}\right)-\mathcal{R}\left(W, D_{\text {out }}\right)\right|}{\left|f\left(W, D_{\text {out }}\right)\right|} .
$$

In both cases, $5 \times 6$ and $3 \times 2$ samples of LF and HF are considered as shown in Figure 2 . Then using the proposed technique a rational model is obtained for the L and Q. Figure 3 (a) and (b) plots the $\mathrm{L}$ and Q respectively on a denser grid of $12 \times 20$ and is validated with HF data on a similar grid. The relative error (12) for L and Q is plotted in Figure 4 (a) and (b) respectively and can be seen that the model accurately captures the true underlying system. The evolution of the normalized root-mean-square error (NRMSE) is compared in terms of iteration count in Figure 5. The NRMSE for $K_{v}$ validation points is defined as,

$$
N R M S E=\frac{\sqrt{\frac{\sum_{k=1}^{K v}\left(f\left(W_{k}, D_{\text {out }}\right)-\mathcal{R}\left(W_{k}, D_{\text {out }}\right)\right)^{2}}{K_{v}}}}{\max \left(f\left(W, D_{\text {out }}\right)\right)-\min \left(f\left(W, D_{\text {out }}\right)\right)} .
$$

To illustrate the correlation of the multi-fidelity model with the samples considered for modeling, the Spearman Rank Order Correlation Coefficient (SROCC) and the Pearson Linear Correlation Coefficient (PLCC) is studied for both cases. In Table 2, the multi-fidelity model is

\footnotetext{
${ }^{1}$ www.eesof.com, Agilent Technologies EEsof EDA, Santa Rosa, CA.
} 


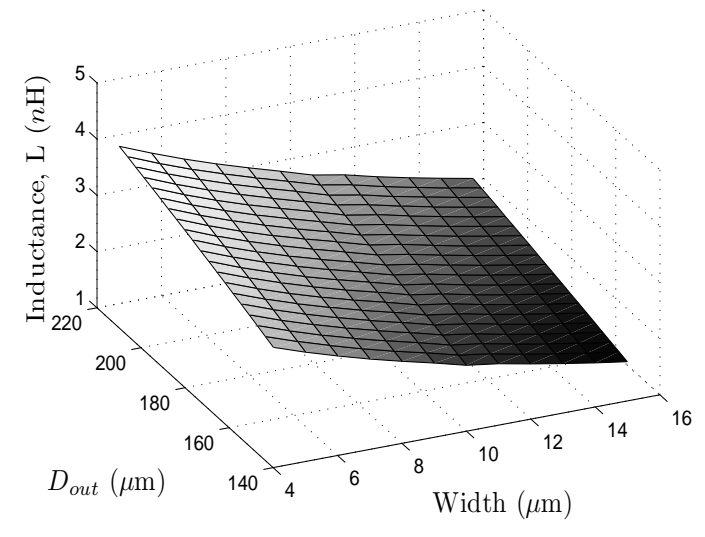

(a) Multi-fidelity model for Inductance

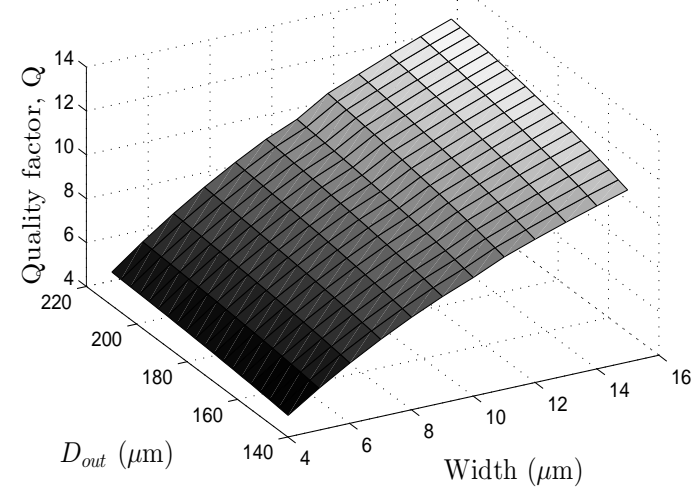

(b) Parametric rational model for Quality factor, Q.

Figure 3: (a) and (b) plots the model obtained for inductance (L) and quality factor (Q) using the rational function.

compared with the HF and LF training samples that were used for the modeling as shown in Figure 2. It can be seen that the samples and the model have a strong linear relationship as the SROCC and PLCC is 0.99. The model is also compared with the HF data used for validation and is seen that the SROCC and PLCC is equal to 0.99, which again infers that the model response is strongly correlated with the true $\mathrm{L}$ and $\mathrm{Q}$ values, see Figure 6 . Table 2 also gives the values of the NRMSE (13) to assess the multi-fidelity model accuracy for both L and Q.

Table 2: CORRELATION OF MULTI-FIDELITY MODEL WITH THE ACTUAL SAMPLES.

\begin{tabular}{l|lll|lll}
\hline \multirow{2}{*}{ Data set } & \multicolumn{3}{|c}{ For Inductance (L) } & \multicolumn{3}{c}{ For Quality Factor (Q) } \\
\cline { 2 - 8 } & NRMSE & SROCC & PLCC & NRMSE & SROCC & PLCC \\
\hline High-fidelity training & 0 & 1 & 1 & 0 & 1 & 1 \\
Low-fidelity training & 0.092 & 0.99 & 0.99 & 0.08 & 0.99 & 0.99 \\
Validation (high-fidelity) & 0.012 & 0.99 & 0.99 & 0.003 & 0.99 & 0.99 \\
\hline
\end{tabular}

Thus, the proposed technique generates a rational model from the LF and HF data which is more accurate than a model built using either the sparse HF samples or dense LF samples only. Also the model is built at a reduced cost when compared to a model that is built from a larger amount of HF samples only (see Table 3), leading to a speedup of 21 times.

\section{CONCLUSIONS}

A novel approach for multivariate modeling of systems using a combination of variable fidelity simulations has been proposed. The algorithm first makes use of multidimensional barycentric interpolation for computing a rational model with the HF data, and then the coefficients of this model are iteratively calculated using the (calibrated) LF data in least-square sense. It is shown that rational function models can be built at a reduced cost by combining different types of data. The effectiveness of the technique is illustrated using a numerical result. 


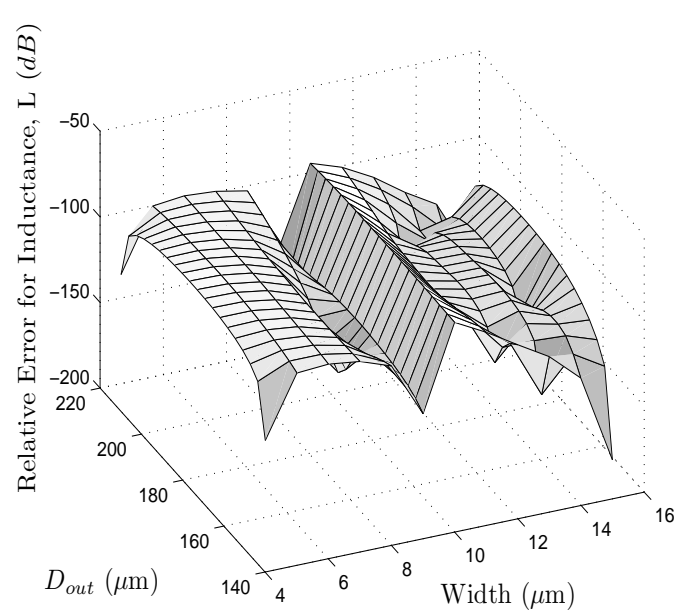

(a) Relative Error for Inductance, L

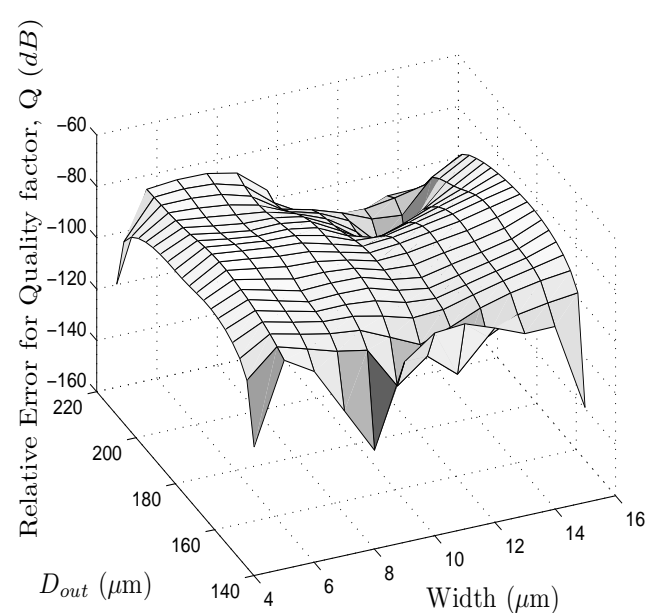

(b) Relative Error for Quality factor, Q

Figure 4: (a) and (b) plots the relative error of the model with the original model which is the high-fidelity data.

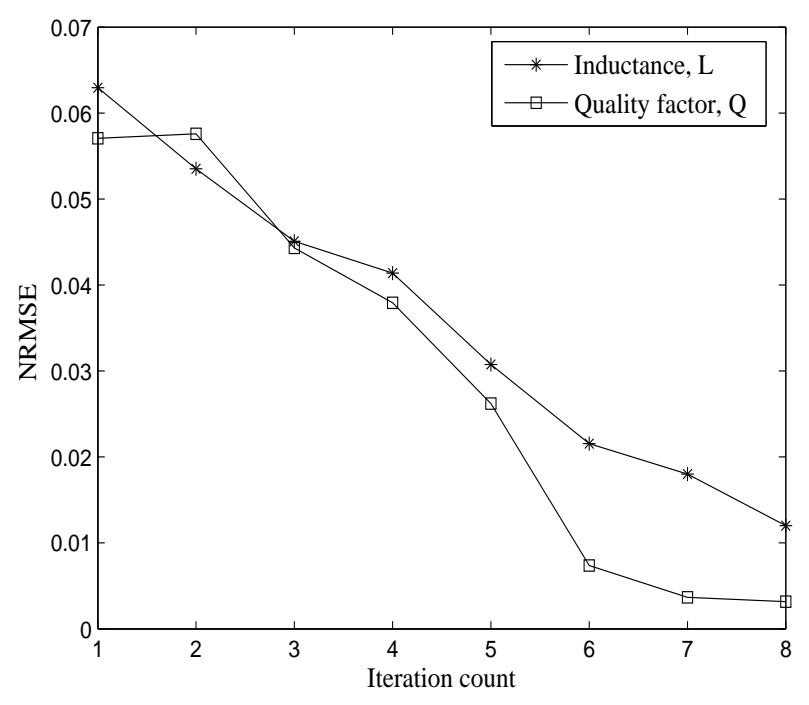

Figure 5: Evolution of NRMSE in terms of iteration count t.

\section{ACKNOWLEDGMENTS}

This research has been funded by the Fund for Scientific Research in Flanders (FWOVlaanderen) and the Interuniversity Attraction Poles Programme BESTCOM initiated by the Belgian Science Policy Office.

\section{REFERENCES}

[1] P. Craig, M. Goldstein, A. Seheult, and J. Smith, "Constructing partial prior specifications for models of complex physical systems.," Journal of the Royal Statistical Society Series D (The Statistician), vol. 47, no. 1, pp. 37-53, 1998. 

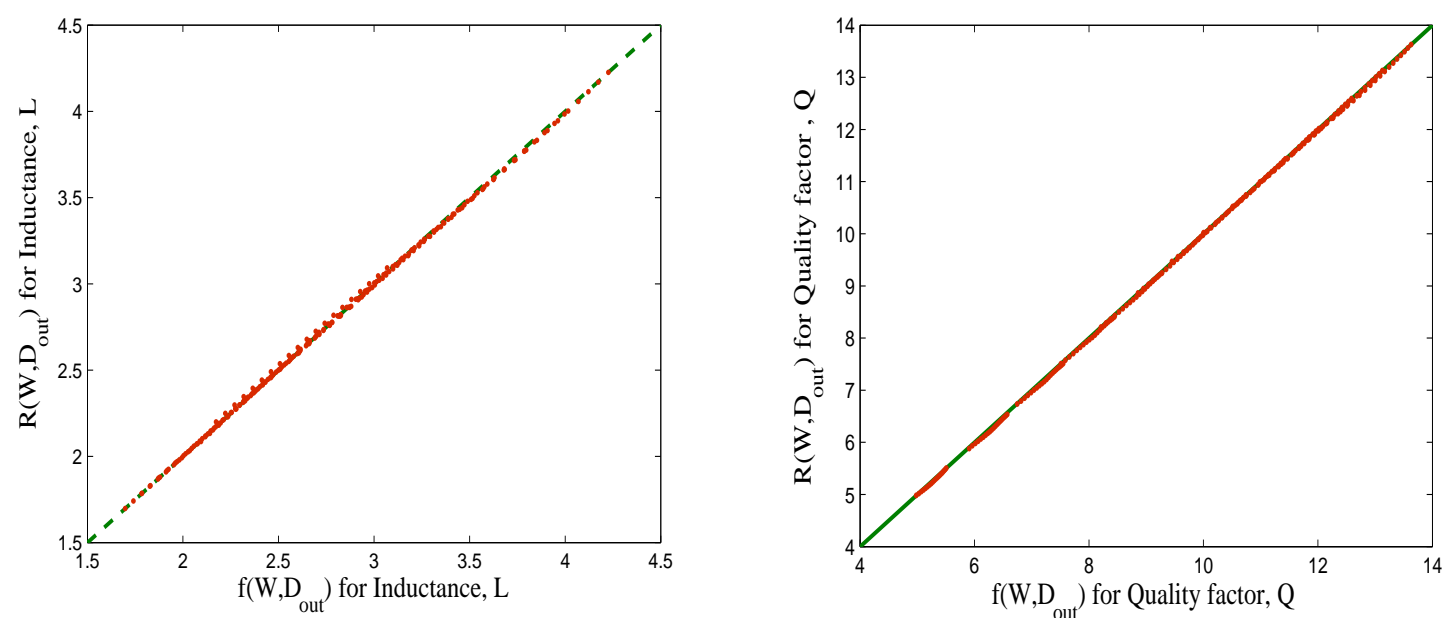

Figure 6: Plots the observed value to the predicted value for the inductance (L) and quality factor (Q).

Table 3: COMPUTATION TIMES.

\begin{tabular}{lll}
\hline Data set & samples & CPU time (s) \\
\hline Calculation of LF training samples & $5 \times 6$ & 0.903 \\
Calculation of HF training samples & $3 \times 2$ & 1.525 \\
Rational function modeling & & 0.267
\end{tabular}

Evaluation of multi-fidelity model over a dense grid $\quad 12 \times 20 \quad 0.209$

Total 2.904

Speedup 21

Calculation of HF data over a dense grid

$12 \times 20 \quad 60.984$

[2] M. Bakr, J. Bandler, K. Madsen, and J. Søndergaard, "An Introduction to the Space Mapping Technique," Optimization and Engineering, vol. 2, no. 4, pp. 369-384, 2001.

[3] D. Huang, T. Allen, W. Notz, and R. Miller, "Sequential kriging optimization using multiple-fidelity evaluations," Structural and Multidisciplinary Optimization, vol. 32, no. 5, pp. 369-382, 2006.

[4] M. C. Kennedy and A. O'Hagan, "Predicting the output from a complex computer code when fast approximations are available," Biometrika, vol. 87, pp. 1-13, Mar. 2000.

[5] I. Couckuyt, F. Declercq, T. Dhaene, H. Rogier, and L. Knockaert, "Surrogate-based infill optimization applied to electromagnetic problems," International Journal of RF and Microwave Computer-Aided Engineering, vol. 20, no. 5, pp. 492-501, 2010.

[6] J. Willems and J. Polderman, Introduction to Mathematical Systems Theory: A Behavioral Approach. NewYork: Springer-Verlag, 1998. 
[7] E. Safe and R. S. Varga, Padé and Rational Approximation: Theory and Applications. NewYork: Academic Press Inc., 1977.

[8] Y.-1. F. Chiang, "A modified remes algorithm," SIAM J. Sci. Stat. Comput., vol. 9, pp. 1058-1072, Nov. 1988.

[9] C. K. Sanathanan and J. Koerner, "Transfer function synthesis as a ratio of two complex polynomials," IEEE Transactions on Automatic Control, vol. 8, no. 1, pp. 56-58, 1963.

[10] B. Gustavsen and A. Semlyen, "Rational approximation of frequency domain responses by vector fitting," IEEE Transaction on Power Delivery, vol. 14, pp. 1052-1061, Jul. 1999.

[11] R. Lehmensiek and P. Meyer, "Creating accurate multivariate rational interpolation models of microwave circuits by using efficient adaptive sampling to minimize the number of computational electromagnetic analyses," IEEE Transactions on Microwave Theory and Techniques, vol. 49, pp. 1419-1430, Aug 2001.

[12] A. Lamecki, P. Kozakowski, and M. Mrozowski, "Efficient implementation of the cauchy method for automated cad-model construction," IEEE Microwave and Wireless Components Letters, vol. 13, pp. 268-270, July 2003.

[13] D. Deschrijver, T. Dhaene, and D. De Zutter, "Robust parametric macromodeling using multivariate orthonormal vector fitting," IEEE Transactions on Microwave Theory and Techniques, vol. 56, no. 7, pp. 1661-1667, 2008.

[14] A. Lamecki, L. Balewski, and M. Mrozowski, "Adaptive cad-model construction schemes," IEEE Transactions on Magnetics, vol. 45, pp. 1538-1541, March 2009.

[15] C. Schneider and W. Werner, "Some new aspects of rational interpolation," Mathematics of Computation, vol. 47, no. 175, pp. pp. 285-299, 1986.

[16] L. Knockaert, "A simple and accurate algorithm for barycentric rational interpolation," IEEE Signal Processing Letters, vol. 15, pp. 154-157, 2008.

[17] V. V. Toropov, F. V. Keulen, V. L. Markine, and L. F. Alvarez, "Multipoint approximation based on response surface fitting: a summary of recent developments," First ASMKO UK/ ISSMO., pp. 371-380, 1999.

[18] R. T. Haftka, "Combining global and local approximations.," AIAA Journal, vol. 29, no. 9, pp. 1523-1525, 1991.

[19] K. J. Chang, R. T. Haftka, G. L. Giles, and P. J. Kao, "Sensitivity-based scaling for approximating structural response.," Journal of Aircraft, vol. 30, no. 2, pp. 283-287, 1993.

[20] D. Deschrijver and T. Dhaene, "Stability and passivity enforcement of parametric macromodels in time and frequency domain," IEEE Transactions on Microwave Theory and Techniques, vol. 56, pp. 2435-2441, Nov 2008.

[21] E. C. Levy, “Complex-curve fitting," IRE Transactions on Automatic Control, vol. AC-4, pp. 37-43, May 1959. 
[22] D. Deschrijver, B. Gustavsen, and T. Dhaene, "Advancements in iterative methods for rational approximation in the frequency domain," IEEE Transactions on Power Delivery, vol. 22, no. 3, pp. 1633-1642, 2007.

[23] B. Gustavsen, "Improving the pole relocating properties of vector fitting," Power Delivery, IEEE Transactions on, vol. 21, no. 3, pp. 1587-1592, 2006.

[24] B. D. O. Anderson and S. Vongpanitlerd, Network Analysis and Synthesis: A Modern Systems Theory Approach. NewYork: Dover Publications, 2006. 